\title{
Characterization of bafilomycin biosynthesis in Kitasatospora setae KM-6054 and comparative analysis of gene clusters in Actinomycetales microorganisms
}

\author{
Ayako Nara ${ }^{1,3}$, Takuya Hashimoto ${ }^{2,3}$, Mamoru Komatsu $^{1}$, Makoto Nishiyama ${ }^{2}$, Tomohisa Kuzuyama ${ }^{2}$ \\ and Haruo Ikeda ${ }^{1}$
}

Bafilomycins $A_{1}, C_{1}$ and $B_{1}$ (setamycin) produced by Kitasatospora setae KM-6054 belong to the plecomacrolide family, which exhibit antibacterial, antifungal, antineoplastic and immunosuppressive activities. An analysis of gene clusters from $K$. setae KM-6054 governing the biosynthesis of bafilomycins revealed that it contains five large open reading frames (ORFs) encoding the multifunctional polypeptides of bafilomycin polyketide synthases (PKSs). These clustered PKS genes, which are responsible for bafilomycin biosynthesis, together encode 11 homologous sets of enzyme activities, each catalyzing a specific round of polyketide chain elongation. The region contains an additional 13 ORFs spanning a distance of $73287 \mathrm{bp}$, some of which encode polypeptides governing other key steps in bafilomycin biosynthesis. Five ORFs, BfmB, BfmC, BfmD, BfmE and BfmF, were involved in the formation of methoxymalonyl-acyl carrier protein (ACP). Two possible regulatory genes, bfmR and $b f m H$, were found downstream of the above genes. A gene-knockout analysis revealed that BfmR was only a transcriptional regulator for the transcription of bafilomycin biosynthetic genes. Two genes, $b f \mathrm{fm}$ and $\mathrm{bfmJ}$, were found downstream of $b f \mathrm{fH}$. An analysis of these gene-disruption mutants in addition to an enzymatic analysis of $\mathrm{Bfml}$ and $\mathrm{BfmJ}$ revealed that $\mathrm{BfmJ}$ activated fumarate and $\mathrm{Bfml}$ functioned as a catalyst to form a fumaryl ester at the $\mathrm{C} 21$ hydroxyl residue of bafilomycin $A_{1}$. A comparative analysis of bafilomycin gene clusters in K. setae KM-6054, Streptomyces Iohii JCM 14114 and Streptomyces griseus DSM 2608 revealed that each ORF of both gene clusters in two Streptomyces strains were quite similar to each other. However, each ORF of gene cluster in K. setae KM-6054 was of lower similarity to that of corresponding ORF in the two Streptomyces species.

The Journal of Antibiotics (2017) 70, 616-624; doi:10.1038/ja.2017.33; published online 15 March 2017

\section{INTRODUCTION}

The natural products bafilomycins ${ }^{1,2}$ (Figure 1), $\mathrm{A}_{1}$ and $\mathrm{B}_{1}$ $\left(\right.$ setamycin ${ }^{3}$ ), as well as concanamycin $\mathrm{A}$, ${ }^{4}$ were discovered using specific biological screening programs from various Actinomycetales microorganisms and belong to the plecomacrolide family due to their unusually folded side chain. ${ }^{5}$ The unique and most interesting structural element of the plecomacrolide family is the stereospecific formation of an intra-molecular hemiacetal within a long side chain. The resulting tetrahydropyrane ring and macrocyclic lactone are linked by a $\mathrm{C}_{3}$ spacer and hydrogen bonding system. ${ }^{6}$ The members within the families differ in the substituents, which are attached to the hemiacetal portion (carbohydrates as in concanamycin A, fumaric acid as in viranamycin $A^{7}$ and bafilomycin $C_{1}$ or fumaric acid derivatives as in virustomycin $\mathrm{A}^{8}$ and bafilomycin $\mathrm{B}_{1}$ ).

Bafilomycins exhibit antibacterial (active against Gram-positive bacteria), antifungal, antineoplastic and immunosuppressive activities. ${ }^{2}$ Bafilomycins $A_{1}$ and $B_{1}$ are very potent and specific inhibitors of vacuolar-type $\mathrm{H}^{+}$-ATPase, which has led to a lot of interest in these metabolites. ${ }^{9}$ Bafilomycins prevent the re-acidification of synaptic vesicles once they have undergone exocytosis. Vacuolar-type ATPases are involved in bone resorption processes in osteoclasts and thus these macrolides have potential in the treatment of osteoporosis. ${ }^{10,11}$

Bafilomycins were previously reported to be produced by various Actinomycetales microorganisms including $K$. setae $\mathrm{KM}-6054,{ }^{3}$ K. griseola MK730-N6, ${ }^{12}$ K. cheerisanensis KCTC $2395,{ }^{13}$ S. lohii JCM $14114,{ }^{14}$ S. griseus DSM $2608^{15}$ and Streptomyces sp. M10. ${ }^{16}$ Therefore, the evolution of gene clusters for bafilomycin biosynthesis has been attracting interest, because biosynthetic gene clusters are distributed among the different genera of Actinomycetales microorganisms. Furthermore, modification steps in bafilomycin biosynthesis have not yet been examined in detail.

The engineered biosynthesis of altered bafilomycin derivatives will enhance the potency and spectrum of activity of these compounds and

${ }^{1}$ Kitasato Institute for Life Sciences, Kitasato University, Kanagawa, Japan and ${ }^{2}$ Biotechnology Research Center, University of Tokyo, Tokyo, Japan

${ }^{3}$ These authors contributed equally to this work.

Correspondence: Professor H Ikeda, Kitasato Institute for Life Sciences, Kitasato University, 1-15-1 Kitasato, Minami-ku, Sagamihara, Kanagawa 252-0373, Japan.

E-mail: ikeda@ls.kitasato-u.ac.jp

Dedicated to Professor Satoshi Ōmura for his numerous contributions to the field of natural products.

Received 18 October 2016; revised 4 January 2017; accepted 1 February 2017; published online 15 March 2017 
reducing their toxicities. Therefore, we herein investigated the organization of bafilomycin biosynthetic genes in K. setae KM-6054 and performed a comparative analysis on gene clusters in bafilomycinproducing Actinomycetales microorganisms.

\section{MATERIALS AND METHODS}

\section{Bacterial strains and growth conditions}

Bafilomycin-producing $K$. setae $\Delta k s b A^{17}$ was used as a parent strain in the present study (designated as the 'wild-type' strain), because $\Delta k s b A$ mutants produce more bafilomycins than the type strain KM-6054. All K. setae strains were grown in trypticase soy broth (Nissui Pharmaceutical Co., Ltd, Tokyo, Japan) at $28^{\circ} \mathrm{C}$ for 2 days and mycelia were washed with sterile water. Washed mycelia were suspended in sterile $25 \% \mathrm{v} / \mathrm{v}$ glycerol and the suspension was stored at $-80^{\circ} \mathrm{C}$. A small block of the frozen mycelial suspension of $K$. setae was used to inoculate a $50 \mathrm{ml}$ test tube containing $10 \mathrm{ml}$ of vegetative medium ${ }^{18}$ and the culture was allowed to grow with reciprocal shaking at $28^{\circ} \mathrm{C}$ for 2 days. A $0.1 \mathrm{ml}$ portion of the vegetative culture was used to inoculate a $125 \mathrm{ml}$ flask containing $10 \mathrm{ml}$ of production medium SSY (25 g of soluble starch, $15 \mathrm{~g}$ of soy flour, $2 \mathrm{~g}$ of yeast extract and $4 \mathrm{~g}$ of $\mathrm{CaCO}_{3}$ in 11 of deionized water adjusted to $\mathrm{pH}$ 7.0) or YD (4 g of yeast extract, $10 \mathrm{~g}$ of malt
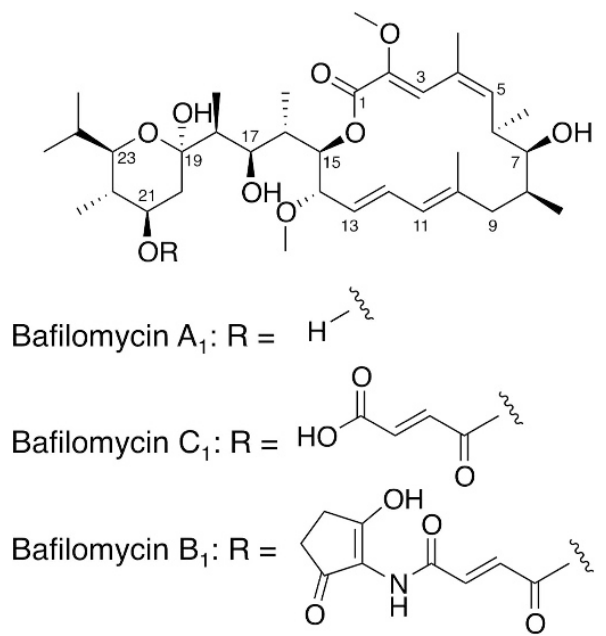

Figure 1 Structures of bafilomycins $A_{1}, C_{1}$ and $B_{1}$ (setamycin). extract and $40 \mathrm{~g}$ of dextrin in 11 of deionized water adjusted to $\mathrm{pH} 7.0$ ) and the culture was allowed to grow with shaking at $28^{\circ} \mathrm{C}$ for 5 days. $K$. setae mainly produced bafilomycins $A_{1}$ and $C_{1}$ in SSY medium and bafilomycin $B_{1}$ in YD medium.

Escherichia coli DH5 $\alpha$ and GM2929 hsdS::Tn10 18 were grown in Luria agar ( $10 \mathrm{~g}$ of tryptone, $5 \mathrm{~g}$ of yeast extract, $5 \mathrm{~g}$ of $\mathrm{NaCl}$ and $15 \mathrm{~g}$ of agar in 11 of deionized water adjusted to $\mathrm{pH}$ 7.5) with appropriate antibiotics. The construction of the gene-disruption mutants of $K$. setae was described in Supplementary Information.

\section{Transformation of $K$. setae using recombinant DNA by electroporation}

K. setae $\Delta k s b A$ was inoculated into a $500 \mathrm{ml}$ flask containing $100 \mathrm{ml}$ of YMG medium ( $4 \mathrm{~g}$ of yeast extract, $10 \mathrm{~g}$ of malt extract and $4 \mathrm{~g}$ of glucose in $1 \mathrm{l} \mathrm{of}$ deionized water adjusted to $\mathrm{pH} 7.0)$ at $28^{\circ} \mathrm{C}$ for 3 days with shaking at 180 r.p.m. After cultivation, submerged spores ${ }^{19}$ were isolated by filtration using sterile Whatman No. 1 filter paper. The filtrate was centrifuged at 3000 r.p.m. for $10 \mathrm{~min}$ to sediment the submerged spores. The sedimented submerged spores were then washed twice with sterile water $(100 \mathrm{ml}$ each). Washed submerged spores were suspended in $0.8 \mathrm{ml}$ of sterile $10 \% \mathrm{v} / \mathrm{v}$ glycerol. An unmethylated recombinant plasmid prepared in E. coli GM2929 hsdS::Tn10 was added to $0.05 \mathrm{ml}$ of the submerged spore suspension. The mixture was placed into an electroporation cuvette (1 mm gap) and electroporation was performed at $1.75 \mathrm{kV}(100 \Omega$ and $25 \mu \mathrm{F})$. One milliliter of trypticase soy broth was immediately added and the suspension was incubated with shaking at $30^{\circ} \mathrm{C}$ for $3 \mathrm{~h}$. After incubation, the suspension was diluted and spread onto $\mathrm{YMG}^{++}$(4 g of yeast extract, $10 \mathrm{~g}$ of malt extract, $4 \mathrm{~g}$ of glucose and $20 \mathrm{~g}$ of agar in 1 liter of deionized water adjusted to $\mathrm{pH}$ 7.0. After autoclaving, $1 \mathrm{M}$ $\mathrm{MgCl}_{2}$ and $1 \mathrm{M} \mathrm{CaCl}_{2}$ were added to make 10 and $8 \mathrm{~mm}$, respectively) plates containing $25 \mu \mathrm{g} \mathrm{ml}^{-1}$ of apramycin. The plates were incubated at $28^{\circ} \mathrm{C}$ for 5 days.

\section{Detection of metabolites from the culture}

After cultivation, the culture was separated by centrifugation at 3000 r.p.m. for $10 \mathrm{~min}$. The supernatant was discarded and sedimented mycelia were extracted with $5 \mathrm{ml}$ of acetone for $15 \mathrm{~min}$. After mycelia were sedimented by centrifugation at 3000 r.p.m. for $10 \mathrm{~min}$, the supernatant was collected and evaporated under reduced pressure to remove acetone. The residue was dissolved in $0.2 \mathrm{ml}$ of chloroform. After insoluble material was removed by filtration, the organic extract was stored at $-20^{\circ} \mathrm{C}$ before a HPLC analysis. Each acetone extract of mycelia was analyzed by HPLC (ELITE LaChrom system, Hitachi Hi-

a



Figure 2 Organization of ORFs in gene clusters for bafilomycin biosynthesis in (a) K. setae KM-6054, (b) S. Iohii JCM 14114 and (c) S. griseus DSM 2608. Red-colored arrows indicate genes encoding type-I PKSs and a discrete thioesterase (editing function), magenta-colored arrows indicate genes encoding the biosynthesis of methoxymalonyl-ACP, blue-colored arrows indicate genes involved in post-polyketide modifications for the conversion from bafilomycin $\mathrm{C}_{1}$ to $B_{1}$, green-colored arrows indicate genes involved in post-polyketide modifications for the conversion from bafilomycin $A_{1}$ to $C_{1}$, yellow-colored arrows indicate genes encoding the transcriptional regulator for the expression of biosynthetic genes and open arrows indicate genes encoding unknown functions. A full color version of this figure is available at The Journal of Antibiotics journal online. 
Table 1 Deduced functions of ORFs involving bafilomycin biosynthesis in K. setae KM-6054, S. griseus DSM 2608 and S. lohii JCM 14114

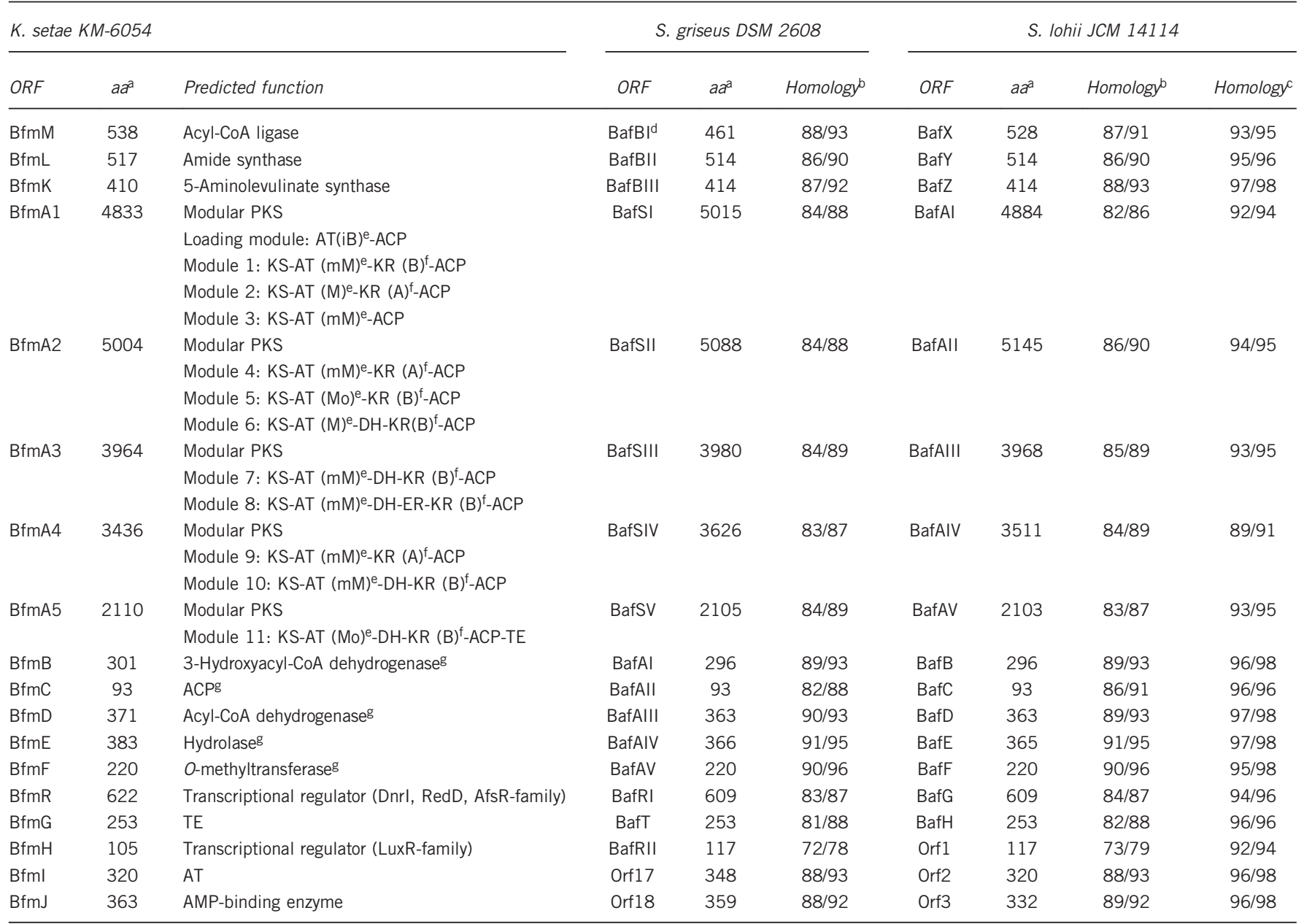

Abbreviations: A, A-type; ACP, acyl carrier protein; AT, acyltransferase; B, B-type; DH, dehydratase; ER, enoyl reductase; iB, isobutyryl-ACP extender; KR, $\beta$-ketoacyl-ACP reductase; KS, $\beta$-ketoacylACP synthase; M, malonyl-ACP extender; mM, methylmalonyl-ACP extender; Mo, methoxymalonyl-ACP extender; ORF, open reading frame; TE, thioesterase.

aNumber of amino acids.

bHomology to the ORF of $K$. setae KM-6054 (\% identity/similarity).

${ }^{c}$ Homology to the ORF of S. griseus DSM 2608 (\% identity/similarity).

dPartial sequence.

epredicted substrate specificity of AT: iB, M, mM anf Mo.

fPredicted stereochemistry type of KR: A and B.

gThese genes were involved in the biosynthesis of methoxymalonyl-ACP.

Technologies Co., Tokyo, Japan) connected to a quadrupole time-of-flight mass spectrometer (Micromass, Q-Tof Ultra API, Waters Co., Milford, MA, USA). The compounds produced were evaluated by a comparison with the mass spectra and ultraviolet absorption of the corresponding authentic samples, bafilomycins $\mathrm{A}_{1}, \mathrm{C}_{1}$ and $\mathrm{B}_{1}$, prepared from $K$. setae $\Delta k s b A$.

\section{In vitro enzymatic reaction of $\mathrm{BfmI}$ and $\mathrm{BfmJ}$}

The expression of $b f m I$ and $b f m J$, and purification of $\mathrm{BfmI}$ and $\mathrm{BfmJ}$ were described in Supplementary Information. The reaction mixture containing $0.1 \mathrm{ml}$ of $100 \mathrm{~mm}$ HEPES-NaOH (pH 7.5), $5 \mathrm{~mm} \mathrm{MgCl}_{2}, 5 \mathrm{~mm}$ ATP, $5 \mathrm{~mm}$ fumarate, $0.1 \mathrm{~mm}$ bafilomycin $\mathrm{A}_{1}, 10 \% \mathrm{v} / \mathrm{v}$ glycerol and recombinant $\mathrm{BfmI}$ $\left(1 \mathrm{mg} \mathrm{ml}^{-1}\right)$ and $\mathrm{BfmJ}\left(1 \mathrm{mg} \mathrm{ml}^{-1}\right)$ was incubated for $16 \mathrm{~h}$ at $30^{\circ} \mathrm{C}$. After extraction twice with $0.1 \mathrm{ml}$ of ethyl acetate, phases were separated by centrifugation at 3000 r.p.m. for $5 \mathrm{~min}$. The upper organic phases were combined and dried over anhydrous $\mathrm{Na}_{2} \mathrm{SO}_{4}$. The organic phase was concentrated to dryness and the residue was dissolved in $100 \mu$ l of methanol. A $5 \mu \mathrm{l}$ portion of the extract was subjected to HPLC (UFLC Nexera System, Shimadzu, Kyoto, Japan) connected with time-of-flight mass spectrometer ( $\mathrm{AB}$ Sciex Triple TOF 5600 system).

\section{RESULTS AND DISCUSSION}

A genome analysis of $K$. setae KM-6054 was completed and at least 24 gene clusters were estimated for secondary metabolite biosynthesis. ${ }^{20}$ Of these, four gene clusters, KSE_27200-27290, KSE_65510-65560, KSE_70570-70620 and KSE_73410-73580, contained genes encoding type-I polyketide synthase(s) (PKS). In consideration of the bafilomycin structure, at least 11 modules and 1 loading module were required for polyketide-chain elongation. As the former two gene clusters consisted of one module and four modules with a loading module, respectively, these gene clusters were not involved in bafilomycin biosynthesis. The third gene cluster consisted of non-ribosomal peptide synthetases and type-I PKSs, and was estimated to be involved in factumycin biosynthesis. The last gene cluster showed a strong resemblance in the features of gene organization and deduced amino acid sequences of each open reading frame $(\mathrm{ORF})$ to the biosynthetic gene clusters for bafilomycin of S. lohii JCM $14114^{14}$ and S. griseus DSM $2608^{15}$ (Figure 2 and Table 1). 



Figure 3 Alignment of deduced amino acid sequences of AT and KR domains in conserved regions. A multiple alignment and phylogenetic analysis using the bootstrapping method was performed by CLUSATALW 2.1. Blue-colored AT domains in the module have a methylmalonyl loading function, magenta-colored AT domains have a methoxymalonyl loading function, red-colored AT domains have a malonyl loading function and the green-colored AT domain has a butyryl loading function. Green-colored KR domains in the module have an A-type ketoreductase function and blue-colored KR domains have a B-type ketoreductase function. A full color version of this figure is available at The Journal of Antibiotics journal online.

\section{Region encoding bafilomycin PKS}

We constructed a genomic library of $K$. setae KM-6054 using and an integrating bacterial artificial chromosome (BAC) vector. After the selection of a BAC clone carrying the gene cluster for bafilomycin biosynthesis from the BAC library, the BAC clone carrying the predicted biosynthetic gene cluster for bafilomycin was transformed and expressed in heterologously engineered S. avermitilis SUKA22. The resulting transformants carrying the gene cluster for bafilomycin biosynthesis produced bafilomycins $\mathrm{A}_{1}$ and $\mathrm{B}_{1}{ }^{21}$ The $\mathrm{BAC}$ clone contained the $102.4 \mathrm{~kb}$ region of $K$. setae KM-6054 corresponding to $8331035-8433476 \mathrm{nt}$ of the K. setae KM-6054 genome, which consisted of 48 ORFs (KSE_73350-KSE_73800) including genes involved in bafilomycin biosynthesis $(\mathrm{bfm})$. The $102.4 \mathrm{~kb}$ region described above contained the entire gene cluster for bafilomycin biosynthesis, as transformants produced bafilomycins in the heterologous host. The deduced gene organization within this region is shown in Figure 1 and Table 1. The nucleotide sequence of the biosynthetic gene cluster for bafilomycin and its flanking region was completely elucidated and shown to contain 18 ORFs spanning a distance of $73287 \mathrm{bp}$. This size was sufficiently large to accommodate the 11 extension modules and 1 loading module anticipated for the bafilomycin PKS. The amino acid sequences of the deduced products encoded by these genes were analyzed by comparing them with the amino acid sequences of known type-I PKSs. The presumptive functional analysis of bafilomycin PKSs was based on a comparison with known modular PKS polypeptides. Five genes, bfmA1-A5, encoding a modular PKS were identified. The 11 modules encoding bafilomycin PKSs were transcribed in 1 direction. Five ORFs encoded the multifunctional type-I PKSs: BfmA1 (4821 aa) containing the loading and the first three modules was required for polyketide chain extension, BfmA2 (5004 aa) containing the next three modules with the activities was needed to continue chain elongation up to C11, BfmA3 (3951 aa) containing two modules with activities was needed to continue chain elongation up to C7, BfmA4 (3441 aa) containing two modules with activities was needed to continue chain elongation up to C3 and BfmA5 (2103 aa) containing one module was needed to complete the polyketide-derived bafilomycin backbone. Five large polypeptides contributed 53 catalytic domains.

The first and last modules were encoded unique domains similar to known PKS modules. In contrast to almost PKSs, the N-terminal motifs of the first loading module consisted of non-functional $\beta$-ketoacyl-ACP synthase (KS), acyltransferase (AT), and ACP domains. The non-functional KS of the loading module in macrolide PKSs was enzymatically non-functional for the condensation step, because a critical cysteine residue in the invariant sequence -CSSSLwhich is highly conserved among functional KS domains, was replaced by another amino acid. ${ }^{22}$ Chain initiation on the first loading module of bafilomycin PKS required initial AT and ACP domains to load the starting acyl group onto the PKS. This loading AT-ACP didomain was identified at the $\mathrm{N}$ terminus of $\mathrm{BfmA1}$, adjacent to the KS domain of module 1. A similar loading AT-ACP didomain has been reported in erythromycin ${ }^{23}$ and avermectin ${ }^{24}$ PKSs. Thus, all KS domains in bafilomycin PKS modules were functional (Supplementary Figure S1). On the other hand, chain termination on the bafilomycin PKS requires a thioesterase domain to catalyze to the release of the acyl chain from the bafilomycin PKS at the C terminus of BfmA5.

Bafilomycin AT domains showed more sequence variability than the KS and ACP domains (Supplementary Figure S1), which were 
fundamental catalytic domains for polyketide chain elongation. Malonyl transferring ATs, methylmalonyl transferring ATs and methoxymalonyl transferring ATs have been classified as distinct families based on amino acid sequences and may be distinguished by conserved invariant motifs. It may be possible to predict AT substrate specificity based on the primary amino acid sequence of each AT domain (Figure 3). A multiple alignment and phylogenetic analysis was applied to compare bafilomycin ATs with those from known PKSs (Supplementary Figure S2). The results obtained showed that modules $1,3,4,7,8,9$ and 10 were classified into the group of ATs using methylmalonyl-CoA as the substrate. On the other hand, the ATs of modules 2 and 6 were classified into the malonyl-specific group, whereas the ATs of modules 5 and 11 were classified into the methoxymalonyl-specific group. The AT of the loading module was slightly different from the above groups and was classified into the group of ATs using monocarboxylic acid CoA (ATs of the loading module in avermectin and erythromycin). The AT presumably used isobutyryl-CoA as a substrate, resulting in the isopropyl side chain at

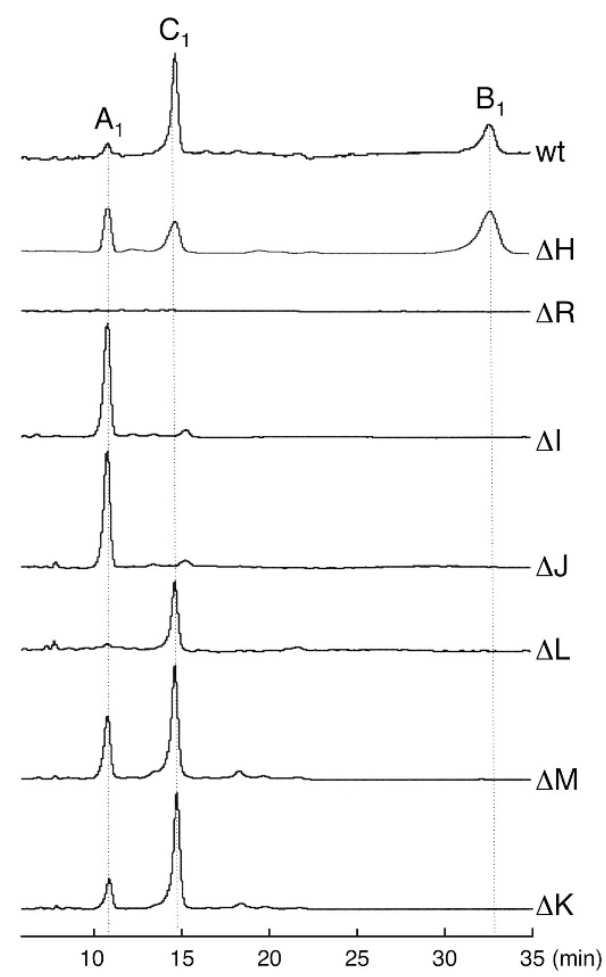

Figure 4 HPLC-MS chromatograms of mycelial extracts from the wild-type $K$. setae and its gene-disruption mutants. Two disruption mutants, bfmH and $\mathrm{bfmR}$, were cultured in YD medium, and other mutants and the wild-type strain were cultured in SSY medium for production. HPLC conditions were as follows: column; CAPCELL PAK $\mathrm{C}_{18}$, MG-II $(3 \mu \mathrm{m}, 2.0 \phi \times 100 \mathrm{~mm})$, mobile phase; $70 \% \mathrm{v} / \mathrm{v}$ acetonitrile in $0.1 \% \mathrm{v} / \mathrm{v}$ trifluoroacetate, flow rate; $0.2 \mathrm{ml} \mathrm{min}^{-1}$, detection at $254 \mathrm{~nm}$. wt indicates the extract from the wild-type strain; $\Delta \mathrm{H}, \Delta \mathrm{R}, \Delta \mathrm{I}, \Delta \mathrm{J}, \Delta \mathrm{L}, \Delta \mathrm{M}$ and $\Delta \mathrm{K}$ are from $b f m H, b f m R$, $\mathrm{bfml}, \mathrm{bfmJ}, \mathrm{bfmL}, \mathrm{bfmM}$ and $\mathrm{bfmK}$ gene-disruption mutants, respectively. $A_{1}, C_{1}$ and $B_{1}$ indicate bafilomycins $A_{1}$ (MW 622.83, m/z $645.82[\mathrm{M}+\mathrm{Na}]^{+}$, $\left.\mathrm{m} / \mathrm{z} 661.93[\mathrm{M}+\mathrm{K}]^{+}\right), \mathrm{C}_{1}\left(\mathrm{MW} 720.89, \mathrm{~m} / \mathrm{z} 743.88[\mathrm{M}+\mathrm{Na}]^{+}, \mathrm{m} / \mathrm{z} 759.98\right.$ $\left.[\mathrm{M}+\mathrm{K}]^{+}\right)$and $\mathrm{B}_{1}\left(\mathrm{MW} 815.99, \mathrm{~m} / \mathrm{z} 838.98[\mathrm{M}+\mathrm{Na}]^{+}, \mathrm{m} / \mathrm{z} 855.08[\mathrm{M}+\mathrm{K}]^{+}\right)$. Each peak in the gene-disruption mutant was also confirmed by MS. Small peaks eluted at approximately $16 \mathrm{~min}$ in $\Delta /$ and $\Delta J$ mutants were identical to bafilomycin $\mathrm{A}_{2}$ (MW 636.87, $\mathrm{m} / \mathrm{z} 659.41[\mathrm{M}+\mathrm{Na}]^{+}, \mathrm{m} / \mathrm{z} 675.39[\mathrm{M}+\mathrm{K}]^{+}$). Bafilomycin $A_{2}$ is a product of bafilomycin $A_{1}$ by methylation of a hydroxyl residue at $\mathrm{C} 19$ under acidic conditions with methanol.
C23. Thus, the substrate prediction was consistent with the structure of the bafilomycin backbone $\mathrm{C} 1$ to $\mathrm{C} 23$.

Dehydratase domains were found in modules $6,7,8,10$ and 11 , whereas the conserved motif sequence-HxxxGxxxxP- was not found in module 6 and the glycine residue in this invariant sequence was replaced by a glutamate residue at position 4102 (Supplementary Figure S1). This replacement of dehydratase in module 6 did not alter the dehydration function because of the presence of olefin at $\mathrm{C} 12$ to C13. A similar type of replacement has been reported in niddamycin PKS. $^{22}$ There was only one enoyl reductase domain in the bafilomycin PKS (Figure 3 and Table 1). An amino acid sequence comparison of the enoyl reductase domain in module 8 with the enoyl reductase domains of known PKSs revealed the presence of the putative $\operatorname{NADP}(\mathrm{H})$ binding motif -LxHxxxGGVG- ${ }^{25,26}$ or $-\mathrm{GxGxxAxxxA-27}$ (Supplementary Figure S1). $\beta$-Ketoacyl-ACP reductase (KR) domains were found in all modules, except for the loading module and module 3. Module 3 was not involved in the reduction at C19 because the module lacked the KR domain. C19 may be a carbonyl group, because the hemiacetal was assumed to be formed by the acetalization of the carbonyl group at C27 and the hydroxyl group at C23. The stereochemistry of $\beta$-hydroxyl carbons at C23, C21, C17, C15 and C7 was controlled by the KR domains of bafilomycin PKSs. KR domains were divided into two groups based on whether they generated an A-type ('S' configuration of the $\beta$-hydroxy residue when $\mathrm{C} 2>\mathrm{C} 4$ ) or B-type (' $\mathrm{R}$ ' configuration of the $\beta$-hydroxy residue when C2 > C4) alcohol stereochemistry. ${ }^{28,29}$ A- and B-type KR sequences were aligned to allow for the identification of residues characteristic of each group. The multiple alignment and phylogenetic analysis was applied to compare bafilomycin KRs. The results obtained showed that the KRs of modules 2, 4 and 9 were classified into the A-type group ('S' configuration), whereas the KRs of modules 1, 5, 6, 7, 8, 10 and 11 were classified into the B-type group ('R' configuration) (Figure 3). The stereochemistries of hydroxyl residues at C7, C15, C17 and C21 in the acyl chain elongated was coincided to corresponding positions of bafilomycin structure. The inter-PKS docking region domain was found in the $\mathrm{N}$ terminus region of PKS, except for PKS containing the first module. Docking region domains similar to those in erythromycin $\mathrm{PKSs}^{30}$ were found in $\mathrm{BfmA} 2$ and $\mathrm{BfmA}$. The shorter sequence was detected in $\mathrm{BfmA4}$, whereas the conserved sequence was not identified in BfmA5 (Supplementary Figure S1). The gene, $b \mathrm{fmG}$ (Figure 2 and Table 1) encoding discrete thioesterase was found downstream of the PKS genes and its gene product may be involved in editing during polyketide chain extension.

\section{ORFs flanking bafilomycin PKS}

The results of the feeding experiments of ${ }^{13} \mathrm{C}$-labeled precursors suggested that the macrocyclic lactone rings of bafilomycins were derived from an isobutyrate starter unit and eleven additional chain extension steps, which involved seven propionate units, two acetate units and two unusual C2 extender units by the chain extension by bafilomycin PKSs. ${ }^{31}$ Two methoxyl groups at the C2 and C4 of bafilomycins were derived from the unusual extender units. The results of the feeding experiments showed that these two methoxy groups originated from glycerol, indicating that the ATs of modules 5 and 11 transferred the methoxyacetyl group by methoxymalonyl-ACP derived from glycerol. Five ORFs, BfmB, BfmC, BfmD, BfmE and $\mathrm{BfmF}$, were found downstream of $b f m A 5$ encoding bafilomycin PKS. These ORFs were involved in the formation of methoxymalonyl-ACP. The generation of methoxylmalonyl-ACP from 1,3-bisphosphoglycerate has already been described in the biosynthesis of other macrolides. ${ }^{32}$ 1,3-Bisphosphoglycerate derived from glycerol through glycerol-3- 
phosphate by glycerol kinase, glycerol-3-phosphate dehydrogenase and glyceraldehyde-3-phosphate dehydrogenase in primary metabolism was acylated on ACP (BfmC) to generate glyceryl-ACP by hydrolase (BfmE). Glyceryl-ACP was oxidized to hydroxymalonyl-ACP by 3-hydroxyacyl-CoA dehydrogenase (BfmB) and acyl-CoA dehydrogenase (BfmD). Hydroxymalonyl-ACP was methylated by $O$-methyltransferase (BfmF) to generate methoxylmalonyl-ACP. Thus, these five ORFs were involved in the biosynthesis of methoxymalonyl-ACP for chain extension (Figure 1 and Table 1). The gene organization of $b f m B$, $b f m C, b f m D, b f m E$ and $b f m F$ was conserved within several pathways. Each of these genes most closely related to their discontinuously distributed counterparts (orf1 $1^{*}, \operatorname{orf}_{2}^{*}, \operatorname{orf}^{*}, \operatorname{orf}^{*}$ and orf $12^{*}$ ) observed in the biosynthetic gene cluster for concanamycin of Streptomyces neyagawaensis ATCC $27449 .{ }^{33}$

The gene cluster for bafilomycin biosynthesis had two possible regulatory genes, $b f m R$ and $b f m H$. Furthermore, two genes, $b f m I$ and $b f m J$, were found downstream of $b f m H$, whereas three genes, $b f m K$, $b f m L$ and $b f m M$, were upstream of $b f m A 1$. These last five ORFs were involved in modifications to the initial macrocyclic lactone (bafilomycin $\mathrm{A}_{1}$ ). In order to confirm the function of each ORF, each gene-knockout mutant was constructed by allelic replacement. The recombinant DNA may be introduced into K. setae by an E. colil Streptomyces conjugation using an IncP-plasmid (RK2) origin transfer. ${ }^{34,35}$ However, we found that the transformation of $K$. setae KM-6054 was improved by electroporation (approximately 10- to 100-fold higher than the conjugation with E. coli and IncP-plasmid). $K$. setae generated submerged spores in the liquid culture and DNA was introduced into the submerged spores by electroporation. Two regulatory genes, $b f m R$ and $b f m H$, were conserved in the motifs of the bacterial transcriptional activator domain (PF03704) and LuxR-family bacterial regulator protein (PF00196), respectively. The disruption mutants of $b f m R$ did not produce bafilomycin, whereas the $b f m H$ disruptant did (Figure 4). This result indicates that BfmR, but not $\mathrm{BfmH}$ has a potential regulatory function in bafilomycin biosynthesis.

As bafilomycin $A_{1}$ was generated by the five bafilomycin PKSs, bafilomycin $\mathrm{B}_{1}$ (and $\mathrm{C}_{1}$ ) may be modified by the other five gene products of $b f m I, b f m J, b f m K, b f m L$ and $b f m M$. $b f m I$ and $b f m J$ genedisruption mutants were unable to produce bafilomycins $\mathrm{C}_{1}$ and $\mathrm{B}_{1}$; these mutants only produced bafilomycin $\mathrm{A}_{1}$ (Figure 4). Other $b f m K-$, $b f m L$ - and $b f m M$-disruption mutants were also unable to produce bafilomycin $B_{1}$. These three gene-disruption mutants produced not only bafilomycin $A_{1}$ but also bafilomycin $C_{1}$ (Figure 4). These results indicate that the former two genes, $b f m I$ and $b f m J$, are involved in the conversion of bafilomycin $A_{1}$ to $C_{1}$. BfmJ may have a role in the activation of fumarate and $\mathrm{BfmI}$ catalyzes the connection of the fumarate moiety from activated fumarate at the C21 hydroxyl residue in the hemiacetal ring. As $b f m K, b f m L$ and $b f m M$ gene-disruption mutants mainly produced bafilomycin $C_{1}$, the three genes were involved in the conversion of bafilomycin $C_{1}$ to $B_{1}$. As $B f m K$ was similar to 5-aminolevulinate synthase (EC 2.3.1.37) in the Shemin pathway, it may catalyze the generation of 5-aminolevulinate from glycine and succinyl-CoA. Genes responsible for the formation of 2-amino-3-hydroxy-cyclopenta-2-enone, which is found in several natural products, including bafilomycin $\mathrm{B}_{1}$, virustomycin $\mathrm{A},{ }^{8}$ reductinomycin. ${ }^{36}$ asukamycin $^{37}$ and ECO-02301, ${ }^{38}$ were found upstream of genes encoding type-I PKS. The formation of 2-amino3-hydroxy-cyclopenta-2-enone from 5-aminolevulinate through 2 steps of an enzymatic reaction has been reported. ${ }^{39}$ Two enzymes, ORF34 (accession AAX98209) and ORF35 (accession AAX98210) of the ECO-02301 producer Streptomyces aizunensis NRRL B-11277 were involved in the formation of 2-amino-3-hydroxy-cyclopenta-2-enone from 5-aminolevulinate and ORF33 (accession AAX98208) catalyzed the connection of 2-amino-3-hydroxy-cyclopenta-2-enone to polyenoic acid in order to generate ECO-02301. The amino acid sequences of these ORFs (ORF34, 35 and 33) were similar to those of BfmK

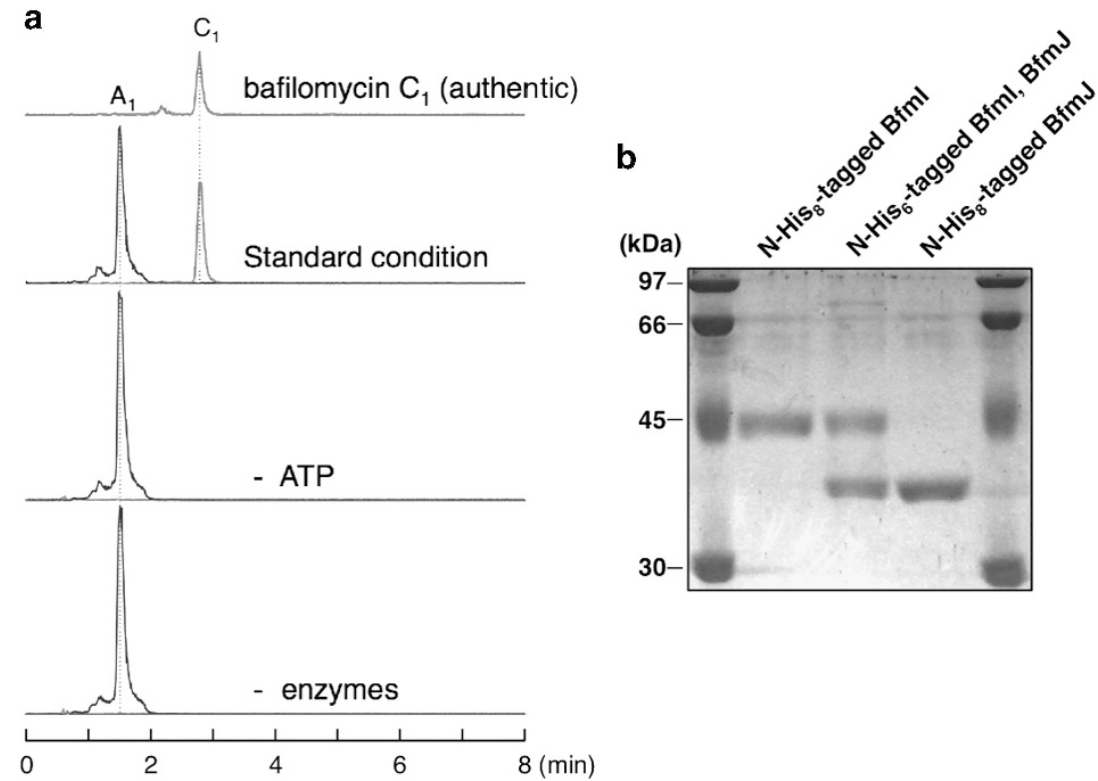

Figure 5 Enzymatic conversion of bafilomycin $A_{1}$ to $C_{1}$ using Bfml and BfmJ. (a) HPLC-MS chromatograms of ethyl acetate extracts of the enzymatic reactions. HPLC conditions were as follows: column: CAPCELL PAK $\mathrm{C}_{18}$, IF ( $\left.2 \mu \mathrm{m}, 2.0 \phi \times 50 \mathrm{~mm}\right)$; mobile phase: $80 \% \mathrm{v} / \mathrm{v} \mathrm{methanol}$ in $0.1 \% \mathrm{v} / \mathrm{v}$ formic acid; flow rate: $0.4 \mathrm{ml} \mathrm{min}{ }^{-1}$. Extracted ion current (XIC) chromatograms are represented at $m / z 645.397 \pm 0.013$ (blue line) corresponding to bafilomycin $A_{1}$ $\left(\mathrm{m} / \mathrm{z} 645.397[\mathrm{M}+\mathrm{Na}]^{+}\right)$and $743.397 \pm 0.013$ (red line) corresponding to bafilomycin $\mathrm{C}_{1}\left(\mathrm{~m} / \mathrm{z} 743.397[\mathrm{M}+\mathrm{Na}]^{+}\right)$, respectively. Each component was verified by authentic samples of bafilomycins $\mathrm{A}_{1}$ and $\mathrm{C}_{1}$. (b) $12.5 \%$ SDS-polyacrylamide gel electrophoresis of purified $\mathrm{N}$-His 8 -tagged Bfml (left), $\mathrm{N}$-His 8 -tagged BfmJ (right) and co-purified $\mathrm{N}-\mathrm{His}_{6}$-tagged Bfml and intact BfmJ (center). A full color version of this figure is available at The Journal of Antibiotics journal online. 


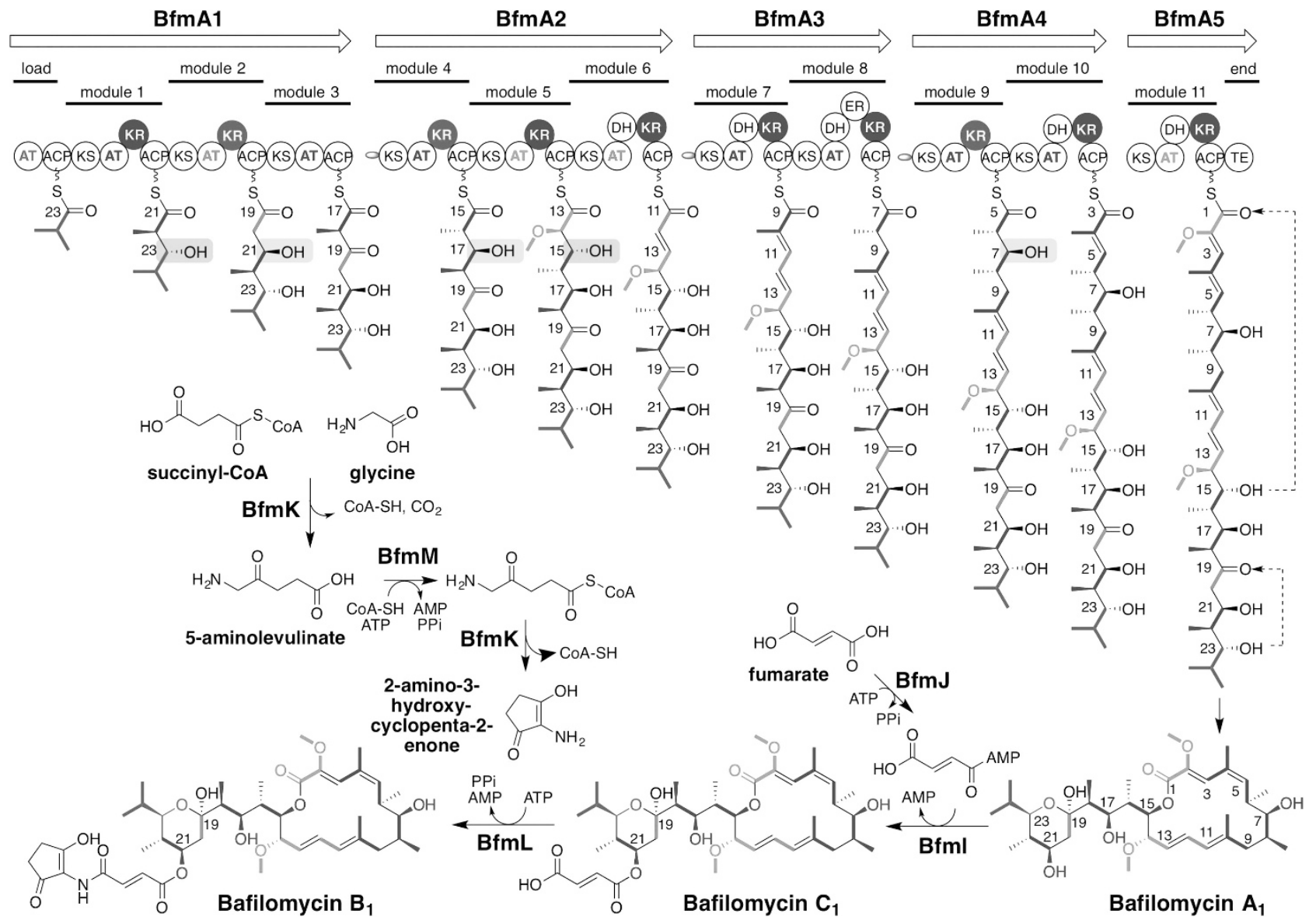

Figure 6 The model for bafilomycin $A_{1}$ formation and predicted domain structure of bafilomycin PKSs and post-polyketide modifications to bafilomycin $A_{1}$ to $B_{1}$ in gene clusters for bafilomycin biosynthesis. Each circle represents an enzymatic domain in the PKS multifunctional polypeptide. Abbreviations are the same as in Table 1. AT domains that are colored in blue; green, magenta and red indicate methylmalonyl-ACP, isobutyryl-ACP, methoxymalonyl-ACP and malonyl-ACP extenders, respectively, and each corresponding acyl chain incorporated is also drawn in the same color. KR domains filled in blue and green indicate B-type and A-type stereochemistries, respectively. The two hydroxyl residues at C23 and C15 are generated by B-type KRs, whereas the three hydroxyl residues at C21, C17 and C7 are by A-type KRs. A full color version of this figure is available at The Journal of Antibiotics journal online.

(67\% identity and 79\% similarity), BfmL (37\% identity and 51\% similarity) and BfmM (30\% identity and $46 \%$ similarity), respectively. 5-Aminolevulinate will be converted to 5-aminolevulinyl-CoA by BfmM. Even though 5-aminolevylinyl-CoA is spontaneously converted to 2,5-piperidinedione due to its instability, it is more easily cyclized to 2-amino-3-hydroxy-cyclopenta-2-enone in the presence of $\mathrm{BfmK}$. Bafilomycin $\mathrm{B}_{1}$ is generated by the connection of 2-amino-3hydroxy-cyclopenta-2-enone to fumaryl residue of bafilomycin $\mathrm{C}_{1}$ by BfmL. Thus, the conversion of bafilomycin $\mathrm{C}_{1}$ to $\mathrm{B}_{1}$ may be performed by BfmK, BfmL and BfmM.

On the other hand, $\mathrm{BfmI}$ and $\mathrm{BfmJ}$ were involved in the conversion of bafilomycin $A_{1}$ to $C_{1}$ based on the intermediates that accumulated in the $b f m I$ and $b f m J$ gene-disruption mutants. The predicted function of ORFs in the biosynthetic gene cluster for bafilomycin were estimated by the statistical analysis of protein families using hidden Markov model (HmmPfam). However, predicted functions of BfmI and $\mathrm{BfmJ}$ were not estimated by HmmPfam. Predicted functions of $\mathrm{BfmI}$ and $\mathrm{BfmJ}$ were analyzed by reversed position-specific BLAST using protein families database. BfmI and BfmJ both had lower similarity to pfam00698 (acyl transferase domain; $22 \%$ identity $/ 32 \%$ similarity) and pfam00501 (AMP-binding enzyme; $22 \%$ identity $/ 29 \%$ similarity), respectively. To elucidate the functions of $\mathrm{BfmI}$ and BfmJ, we overexpressed the $b f m I$ and $b f m J$ genes individually in E. coli as N-terminal $\mathrm{His}_{8}$-tagged proteins and purified the recombinant $\mathrm{BfmI}$ and $\mathrm{BfmJ}$ proteins to near homogeneity. The recombinant $\mathrm{BfmI}$ and $\mathrm{BfmJ}$ were incubated with bafilomycin $\mathrm{A}_{1}$ and fumarate in the presence of ATP and $\mathrm{MgCl}_{2}$, and the reaction mixture was subjected to HPLC-MS analysis. The results revealed the formation of bafilomycin $C_{1}$ only in the presence of ATP (Figure 5a). This enzymatic reaction suggests that $\mathrm{BfmJ}$ uses fumarate and ATP to form fumaryl-AMP transiently and then BfmI forms an ester bond between the carboxyl group of fumarate and the hydroxy residue at the $\mathrm{C} 21$ of bafilomycin $A_{1}$ to generate bafilomycin $C_{1}$. Expected intermediate, fumaryl-AMP, was not detected after incubation of fumarate and ATP with BfmJ in the presence of magnesium ions. Probably fumaryl-AMP will be extremely unstable. As ATP in the reaction mixture was consumed and AMP and pyrophosphate were detected, fumaryl-AMP will be transiently generated from fumarate and ATP with BfmJ. Both $b f m I$ and $b f m J$ genes are located adjacently in the biosynthetic gene cluster and the stop codon of $b f m I$ and the start codon of $b f m J$ are overlapped, suggesting that $b f m I$ was translationally coupled expression with $b f m J$. Thus, we hypothesized that these proteins could interact with each other. To detect the interaction between $\mathrm{BfmI}$ and $\mathrm{BfmJ}$, we constructed a vector for co-expression of $\mathrm{N}$-terminal $\mathrm{His}_{6}$-tagged BfmI and intact BfmJ using the pET-Duet1 vector in E. coli. The co-expressed proteins were purified with Ni-NTA 
Superflow column and analyzed on SDS-polyacrylamide gel electrophoresis. As a result in Figure 5b, the N-terminal His $_{6}$-tagged BfmI and intact $\mathrm{BfmJ}$ were co-purified. This result suggests that both proteins indeed interact with each other and function as hetero-complex. Based on the experimental results obtained, the overall biosynthetic reaction of bafilomycin biosynthesis was estimated in Figure 6 .

Bafilomycins were produced by various Actinomycetales microorganisms including two genera, Streptomyces and Kitasatospora species. Two biosynthetic gene clusters of S. lohii JCM $14114^{14}$ and S. griseus DSM $2608^{15}$ were very similar to each other. As the deduced amino acid sequence similarity of each ORF (Table 1) was in the range of $89 \%$ (identity)/91\% ( similarity) to $97 \%$ (identity)/98\% (similarity), these two gene clusters may have recently evolved. In contrast, the deduced amino acid sequence similarity between K. setae KM-6054 and these two Streptomyces species was lower than that between S. lohii JCM 14114 and S. griseus DSM 2608, in which similarity was between $86 \%$ (identity)/90\% (similarity) and 90\% (identity)/96\% (similarity). The deduced amino acid sequences of the gene products of type-I PKSs and gene products involved in methoxymalonyl-ACP biosynthesis were similar to those of Streptomyces species (between $82 \%$ identity $/ 88 \%$ similarity and $90 \%$ identity $/ 96 \%$ similarity). The deduced amino acid sequence similarities of gene products involved in post-polyketide modifications (BfmI, BfmJ, BfmK, BfmL and BfmM) were also similar to those of Streptomyces species, except for BfmL and BfmM. The ORFs, BfmI and BfmJ, involved in the conversion of bafilomycin $\mathrm{C}_{1}$ to $\mathrm{B}_{1}$ exhibited high similarities to those of Streptomyces species (ranging between $88 \%$ identity/92\% similarity and $88 \%$ identity/93\% similarity). BfmL and BfmM both had lower similarities to those of Streptomyces species (ranging between $86 \%$ identity $/ 90 \%$ similarity and $87 \%$ identity $/ 91 \%$ similarity), whereas BfmK was similar to that of Streptomyces species (ranging between $87 \%$ identity $/ 92 \%$ similarity and $88 \%$ identity $/ 93 \%$ similarity). These three ORFs, BfmL, BfmM and BfmK, were involved in the formation of 2-amino-3-hydroxy-cyclopenta-2-enone and its addition to bafilomycin $\mathrm{C}_{1}$. These results indicate that the 5 -aminolevulinate synthase, BfmK, is highly conserved in Actinomycetales microorganisms. Similarly, BfmI and BfmJ, which are involved in the activation of fumarate and addition of activated fumarate (probably fumaryl-AMP) to the hydroxyl residue at the $\mathrm{C} 21$ of bafilomycin $A_{1}$, may both be highly conserved in Actinomycetales microorganisms.

The genome mining of $K$. setae KM-6054, heterologous expression of gene clusters for bafilomycin biosynthesis, analysis of genedisruption mutants of transcriptional regulators and post-polyketide modification steps have revealed that the region between $8,335,471$ and $8,408,758 \mathrm{nt}$ of $K$. setae KM-6054 contains 18 ORFs spanning a distance of $73287 \mathrm{bp}$. The transcription of bafilomycin biosynthetic genes is controlled by a gene product of $b f m R$ in the gene cluster. Five type-I PKSs generate bafilomycin $A_{1}$, which is converted to bafilomycin $\mathrm{C}_{1}$ by $\mathrm{BfmI}$ using fumarate activated by $\mathrm{BfmJ}$. The final step, the conversion of bafilomycin $C_{1}$ to $B_{1}$, is performed by $B f m L$ using 2-amino-3-hydroxy-cyclopenta-2-enone, which is generated from glycine and succinyl-CoA through 5-aminolevulinate by BfmK and BfmM. The discovery of gene clusters for bafilomycin biosynthesis from many Streptomyces ${ }^{14-16}$ and Kitasatospora ${ }^{3,12,13}$ species suggests that the broad distribution of this gene cluster by horizontal transfer may frequently occur in Actinomycetales microorganisms.

\section{CONFLICT OF INTEREST}

The authors declare no conflict of interest.

\section{ACKNOWLEDGEMENTS}

We thank for S. Kitani and T. Nihira of Osaka University for kindly providing bafilomycin high-yielding producer $K$. setae $\Delta k s b A$ and protocols for conjugative transfer of DNA from E. coli to K. setae.

1 Werner, G., Hagenmaier, H., Albert, K., Kohlshorn, H. \& Drautz, H. The structure of the bafilomycins, a new group of macrolide antibiotics. Tetrahedron Lett. 24 5193-5196 (1983).

2 Werner, G., Hagenmaier, H., Drautz, H., Baumgartner, A. \& Zähner, H. Metabolic products of microorganisms. Bafilomycins, a new group of macrolide antibiotics, production, isolation, chemical structure and biological activity. J. Antibiot. 41, 110-117 (1984).

3 Otoguro, K., Nakagawa, A. \& Omura, S. Setamycin, a 16-membered macrolide antibiotic identification and nematocidal activity. J. Antibiot. 34, 250-252 (1988).

4 Kinashi, H., Someno, K. \& Sakaguchi, K. Isolation and characterization of concanamycins A, B and C. J. Antibiot. 37, 1333-1343 (1984).

5 Bindseil, K. U. \& Zeeck, A. Metabolic products of microorganisms, Prelactones C and B, oligoketides from Streptomyces producing concanamycin and bafilomycins. Helv. Chim. Acta 76, 150-157 (1993).

6 Ley, S. V., Neuhause, D. \& Williams, D. J. A conformational study of elaiphylin by X-ray crystallography and difference ${ }^{1} \mathrm{H}$ NMR methods: observation of a selective sign reversal of the nuclear overhauser effect. Tetrahedron Lett. 23, 1207-1210 (1982).

7 Hayakawa, Y., Takaku, K., Furihata, K., Nagai, K. \& Seto, H. Isolation and structural elucidation of new 18-membered macrolide antibiotics, viranamycins $A$ and $B$. J. Antibiot. 44, 1294-1299 (1991).

8 Omura, S. et al. The structure of virustomycin A. J. Antibiot. 36, 1783-1786 (1983).

9 Browman, E. J., Sibers, A. \& Aldendorf, K. Bafilomycins: a class of inhibitors of membrane ATPases from microorganisms, animal cells and plant cells. Proc. Natl Acad. Sci. USA 85, 7972-7976 (1988).

10 Farina, C. \& Gagliardi, S. Selective inhibition of osteoclast vacuolar $\mathrm{H}^{+}$-ATPase. Curr. Pharm. Design. 8, 2033-2048 (2002).

11 Xu, J., Cheng, T., Feng, H. T., Pavlos, N. J. \& Zheng, M. H. Structure and function of V-ATPases in osteoclasts: potential therapeutic targets for the treatment of osteolysis. Histol. Histopathol. 22, 443-454 (2007).

12 Arens, J. C., Haltli, B. \& Kerr, R. G. Draft genome sequence of Kitasatospora griseola strain MF730-N6, a bafilomycin, terpentecin, and staurosporin producer. Genome Announ. 3, e00208-e00215 (2015).

13 Hwang, J. Y. et al. Draft genome sequence of Kitasatospora cheerisanensis KCTC 2395, which produces plecomacrolide against phytopathogenic fungi. Genome Announ. 2, e00604-e00614 (2014).

14 Zhang, W. et al. Characterization of the bafilomycin biosynthetic gene cluster from Streptomyces lohii. ChemBioChem. 14, 301-306 (2013).

15 Hwang, J. Y., Kim, H. S., Kim, S. H., Oh, H. R. \& Nam, D. H. Organization and characterization of a biosynthetic gene cluster for bafilomycin from Streptomyces griseus DSM 2608. AMB Express 3, 24 (2013).

16 Lee, D. W., Ng, B. G. \& Kim, B. S. Increased valinomycin production in mutants of Streptomyces sp. M10 defective in bafilomycin biosynthesis and branched-chain $\alpha$-keto acid dehydrogenase complex expression. J. Ind. Microbiol. Biotechnol. 42, 1507-1517 (2015).

17 Choi, S. U., Lee, C. K., Hwang, Y. I., Kinoshita, H. \& Nihira, T. Cloning and functional analysis by gene disruption of a gene encoding a $\gamma$-butyrolactone autoregulator receptor from Kitasatospora setae. J. Bacteriol. 186, 3423-3430 (2004).

18 Komatsu, M., Uchiyama, T., Omura, S., Cane, D. E. \& Ikeda, H. Genome-minimized Streptomyces host for the heterologous expression of secondary metabolism. Proc. Nat/ Acad. Sci. USA 107, 2646-2651 (2010).

19 Takahashi, Y., Kuwana, T., Iwai, Y. \& Omura, S. Some characteristics of aerial and submerged spores of Kitasatospora setalba. J. Gen. Appl. Microbiol 30, 223-229 (1984).

20 Ichikawa, N. et al. Genome sequence of Kitasatospora setae NBRC 14216: An evolutionary snapshot of the family Streptomycetaceae. DNA Res. 17, 393-406 (2010).

21 Komatsu, M. et al. Engineered Streptomyces avermitilis host for heterologous expression of biosynthetic gene cluster for secondary metabolites. ACS Synth. Biol. 2, 384-396 (2013).

22 Kakavas, S. J., Katz, L. \& Stassi, D. Identification and characterization of the niddamycin polyketide synthase genes from Streptomyces caelestis. J. Bacteriol. 179 7515-7522 (1997).

23 Donadio, S., Staver, M. J., McAlpine, J. B., Swanson, S. J. \& Katz, L. Modular organization of genes required for complex polyketide biosynthesis. Science 252, 675-679 (1991).

24 Ikeda, H., Nonomiya, T., Usami, M., Ohta, T. \& Omura, S. Organization of the biosynthetic gene cluster for the polyketide anthelmintic macrolide avermectin in Streptomyces avermitilis. Proc. Natl Acad. Sci. USA 96, 9509-9514 (1999).

25 Amy, C. M. et al. Molecular cloning and sequencing of cDNAs encoding the entire rat fatty acid synthase. Proc. Natl Acad. Sci. USA 86, 3114-3118 (1989).

26 Witkowski, J., Rangan, V. S., Randhawa, Z. I., Amy, C. M. \& Smith, S. Structural organization of the multifunctional animal fatty-acid synthase. Eur. J. Biochem. 198, 571-579 (1991).

27 Scrutton, N. S., Berry, A. \& Perham, R. N. Redesign of the coenzyme specificity of a dehydrogenase by protein engineering. Nature 343, 38-43 (1990). 
28 Caffrey, P. Conserved amino acid residues correlating with ketoreductase stereospecificity in modular polyketide synthases. ChemBioChem. 4, 654-657 (2003).

29 Keatinge-Clay, A. T. \& Stroud, R. M. The structure of a ketoreductase determines the organization of the $\beta$-carbon processing enzymes of modular polyketide synthases. Structure 14, 737-748 (2006)

30 Broadhurst, R. W., Nietlispach, D., Wheatcroft, M. P., Leadlay, P. F. \& Weissman, K. J. The structure of docking domains in modular polyketide synthases. Chem. Biol. 10, 723-731 (2003).

31 Schuhmann, T. \& Grond, S. Biosynthetic investigations of the V-type ATPase inhibitors bafilomycin $A_{1}, B_{1}$ and concanamycin A. J. Antibiot. 57, 655-661 (2004).

32 Wenzel, S. C. et al. On the biosynthetic origin of methoxymalonyl-acyl carrier protein, the substrate for incorporation of 'glycelate' units into ansamitocin and soraphen A. J. Am. Chem. Soc. 128, 14325-14336 (2006).

33 Haydock, S. F. et al. Organization of the biosynthetic gene cluster for the macrolide concanamycin A in Streptomyces neyagawaensis ATCC 27449. Microbiology 151, 3161-3169 (2005).
34 Mazodier, P., Petter, R. \& Thompson, C. Intergeneric conjugation between Escherichia coli and Streptomyces species. J. Bacteriol. 171, 3583-3585 (1989).

35 Choi, S. U., Lee, C. K., Hwang, Y. I., Kinoshita, H. \& Nihira, T. Intergeneric conjugal transfer of plasmid DNA from Escherichia coli to Kitasatospora setae, a bafilomycin $\mathrm{B}_{1}$ producer. Arch. Microbiol. 181, 294-298 (2004).

36 Shimizu, K. \& Tamura, G. Reductinomycin, a new antibiotic. II. Structural elucidation by spectroscopic studies. J. Antibiot. 34, 654-657 (1981).

37 Kakinuma, K., Ikekawa, N., Nakagawa, A. \& Omura, S. The structure of asukamycin, a possible shunt metabolite from 3-dehydroquinic acid in the shikimate pathway. J. Am. Chem. Soc. 101, 3402-3404 (1979).

38 McAlpine, J. B. et al. Microbial genomics as a guide to drug discovery and structural elucidation: ECO-02301, a novel antifungal agent, as an example. J. Nat. Prod. 68, 493-496 (2005).

39 Zhang, W., Bolla, M. L., Kahne, D. \& Walsh, C. T. A three enzyme pathway for 2-amino-3-hydroxycyclopent-2-enone formation and incorporation in natural product biosynthesis. J. Am. Chem. Soc. 132, 6402-6411 (2010).

Supplementary Information accompanies the paper on The Journal of Antibiotics website (http://www.nature.com/ja) 